somewhat shorter, but extremely valuable and modern section, on crystal growth.

The crystal growth section provides a concise introduction which would be invaluable to workers in a great many borderline fields, such as metallurgy, material sciences, etc. Discussions are included of what the author calls 'crystal pathology', that is the various disorders and imperfections that arise when departures from precise regularity of the lattice occur. In the crystal chemistry section the author manages to compress an amazing amount of detailed discussion on bonding, structure types, resonance structures, etc. and includes a brief summary of the main features of molecular crystals. The final section provides an authoritative and, again, surprisingly concentrated statement of the main features of crystal physics, dealing with measurements of density, specific heat, hardness, elastic deformation, thermal, electrical and magnetic properties, crystal optics, X-ray electron and neutron scattering.

It is - as the author himself points out in his preface not a book to be read on one's own; it is highly condensed. As a textbook for a comprehensive course in crystallography at third-year undergraduate or M.Sc. level and with adequate laboratory work and supporting lectures and seminars it would be excellent. It is also the kind of book that any experienced crystallographer would wish to have on his reference shelves because of its breadth of coverage and its concise and economical way of presenting information.

The translators have done their work well and are to be congratulated on making this very useful and scholarly work available to English readers.

Department of Physics
University College
P.O. Box 78
Cardiff CF $11 X L$
Wales

C. A. TAYLOR

Neutron physics 2. By G.E.BAcon. Pp.xi+141. London: Wykeham Publications Ltd, 1970. Price $£ 1: 50$

The author of this book is known to every specialist in neutron diffraction for his excellent, and by now classical, textbook on that subject. The present book has a much wider scope and is written for the non-specialist. Dr Bacon has, with the assistance of an experienced schoolmaster, Dr G. R. Noakes, written a very readable account of a wide subject covering neutrons, their discovery, properties, reactions and detection, neutron sources, and application of neutrons for studying static and dynamic properties of solids and liquids. Each chapter contains one or more problems with answers.

The book is written for sixth-formers or undergraduates in English schools and universities. It should, however, be of interest to everybody who is seeking a popular survey of current research and applications of neutron physics. I know of no book that is more suited for technical personnel working with or for a neutron physicist, or for specialists in other fields who want to know what neutron physics is. It is a pleasure to recommend this book.

Institutt for Atomenergi

T. RISTE

Kjeller

Norway
An introduction to mathematical crystallography. By A. W. JASWON. Pp.xi+125. London: Longmans, 1965. Price $£ 1 \cdot 50$.

The reader of this book is introduced to the mathematical notation of crystal-symmetries in four steps: Point group symmetries, space lattices, 'Bravais' space groups (without glide planes and screw axes) and space groups including glide planes and screw axes. These four parts are subdivided into chapters, generally starting from purely geometrical considerations and followed by the corresponding mathematical treatments. At the end of the chapters problems are set, but the book does not contain the solutions.

An introduction to group theory is unfortunately not included. The author refers to numerous texts, but an introduction to the mathematics used, combined with references to relevant literature would probably increase the effectiveness of this book.

It is recommended to crystallographers who will find it a concise but comprehensive text book of geometrical crystallography and corresponding mathematical treatment.

\section{Institut für Kristallographie} und Petrographie / ETH

Sonneggstrasse 5

8006 Zürich

Switzerland

HeInz Schulz

Lattice vibrations. By B. DonOvan and J.F. ANGRESS. Pp.ix + 190. London: Chapman \& Hall, 1971. Price $£ 2.75$.

Lattice dynamics is now a very mature branch of solid state physics, but it has always been difficult to find a text which, for the serious beginner, bridges the gap between brief treatments in general solid-state physics books and comprehensive accounts of various branches of the subject in Summer School publications and review articles. Professor Donovan and Dr Angress are aiming at just such a market in their short monograph on lattice dynamics.

The book confines itself to aspects of the subject explicable in terms of the adiabatic approximation, and derives the most important results one needs to understand the inelastic scattering of neutrons and X-rays, photon-phonon interactions and thermal properties of perfect crystals. There is also a chapter on vibrations of impurities, and a short but informative Bibliography.

The presentation is in general clear, and the contents sensibly chosen; so the book fulfills its primary purpose. For the experienced practitioner of lattice dynamics the collection in one small volume of many useful results may be helpful, but few fresh insights into his discipline will be revealed. A little more space, devoted to putting lattice dynamics into slightly broader perspective, to assessing more critically the current state of the art, and to providing some vision of where lattice dynamics is heading, might have been appreciated by beginner and specialist alike.

Department of Physics
University of York
England

J. A. D. MatTheW 